\title{
The effects of the bioanode on the microbial community and element profile in paddy soil
}

\author{
G. Williamson ${ }^{1} \&$ Z. Chen $^{2}$ \\ ${ }^{1}$ Department of Environmental Science, University of Liverpool, Liverpool, UK \\ ${ }^{2}$ Department of Environmental Science, Xi'an Jiaotong-Liverpool University, Suzhou, P.R. China
}

\begin{abstract}
In paddy soil the reductive dissolution of iron oxide and the availability of organic matter plays an important role in arsenic release under anaerobic conditions. Microbial fuel cells have been shown to reduce organic matter $(\mathrm{OM})$ content and the rate in which this occurs strongly relate to the external resistance applied. In this study we investigated the effects of bioanode operating at different external resistance on the paddy soil microbial community and iron and arsenic concentration. The results show that MFC can be used to reduce soil pore water iron and arsenic concentration and the extent in which this occurs depend on the external resistance applied. The MFC is able to mitigate arsenic release by decreasing organic matter availability. Furthermore, our finding shows that external resistance had a significant influence on the bacterial community composition that develop on the bioanode however only had minimal effect on the community of the bulk soil. These findings suggest that the sMFC can influence the iron and arsenic concentration by reducing OM content and the microbial community that develop in the bioanode vicinity.
\end{abstract}

\section{INTRODUCTION}

Paddy fields are some of the most important farmlands because they are used to cultivate rice; a crop that accounts for more than half of the world's caloric and essential micronutrients intake (Fitzgerald et al., 2009). However, today most paddy fields are contaminated with arsenic (As). Moreover, the increase in arsenic bioavailability in paddy fields has been shown to closely correlate with the redox reactivity of iron oxides. Therefore, investigating iron reduction is crucial to understanding arsenic liberation.

Sediment microbial fuel cells (sMFC) are bioelectrochemical system that has the capacity to simultaneously produce electricity and the removal of organic pollutants (Xu et al., 2015). Recent research have reported that the sMFC bioanode can influence the growth of iron reducing bacteria (Bond et al., 2002; Holmes et al., 2004) and can be used as a substitute for $\mathrm{Fe}$ oxide during exoelectrogens anaerobic respiration. Therefore, the objectives of this study were to examine effects of the bioanode at difference external resistance on iron oxide reductive dissolution and As mobility. Likewise, the changes in microbial community structure were also eluted at the end of 90 days operational period. This study is expected to provide new insight on the role of the SMFC on the liberation of As in soil pore water.

\section{METHODS/EXPERIMENTAL}

Briefly, sMFC was constructed using arsenic pollutant from a rice paddy in Hunan South China. Six different treatments $(50 \mathrm{ohms}, 80 \mathrm{ohms}, 200 \mathrm{ohms}, 1000 \mathrm{ohms}$, $2000 \mathrm{ohms}$ and control) were prepared in triplicates. The control was left at open circuit. A data logger was used to record the voltage between the anode and cathode. Each constructed sMFC was equipped with two soil pore water samplers for collecting pore water, with the lower being adjacent to the anode and the upper being $2 \mathrm{~cm}$ below the sediment-water interface in the bulk soil. All soil pore water total arsenic and iron concentration were determined by inductively coupled plasma emission spectrometry (ICPMS) (NexlonTM 350x, Pekin Elmer, USA) and atomic absorption spectrometry (AAS) (PinAAcle ${ }^{\mathrm{TM}}$ 900, PerkinElmer, USA), respectively, on day 0, 20, $30,40,50$ and 90 . Loss on ignition (LOI) carbon was determined by heating $10 \mathrm{~g}$ of soil at $550^{\circ} \mathrm{C}$ for $4 \mathrm{hrs}$ in a muffle furnace. The microbial community was analyzed after 90 days of operation using 16S rRNA analysis method. The DNA sample will be extracted from the SMFC by using MoBio Laboratories Inc.'s Powersoil DNA isolation Kit according to the manufacturer's instructions. DNA samples was extracted from two points within each sMFC (bulk soil and the anode associated soil).

\section{RESULTS AND DISCUSSION}

\subsection{Electricity production from $\mathrm{SMFC}$}

The sMFCs were operated at different external resisters of 50, 80, 200, 1000 and $2000 \mathrm{ohms}$ for 90 days. The current of the all sMFC sharply increase during the initial stage regardless of external resistance and reached a maximum current at around 10 days. The highest current produce was observed at an external resistance of $50 \mathrm{ohms}(2.4 \mathrm{~mA})$, followed by those of $80(2.1 \mathrm{~mA}), 200(1.6 \mathrm{~mA})$ and $1000(0.8 \mathrm{~mA})$ ohms. An external load of $2000 \mathrm{ohms}$ produced the lowest current $(0.3 \mathrm{~mA})$. However after ca 50 days all the current output irrespective of external load was approximately the same $(0.15-0.2 \mathrm{~mA})$. The results 


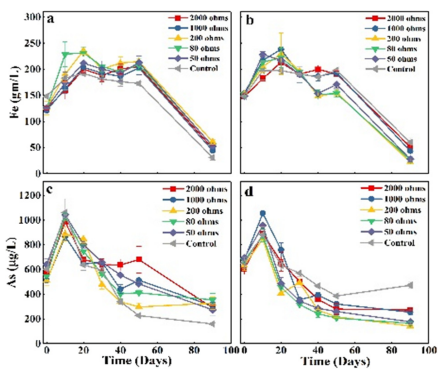

Figure 1. Iron and As variation in soil porewater along with incubation time. $\mathrm{a}$ and $\mathrm{b}$ represent iron concentration in the bulk soil and anode vicinity, respectively; $\mathrm{c}$ and $\mathrm{d}$ represent As concentration in the in the bulk soil and anode vicinity, respectively. The error bars represent standard error of measured concentrations of triplicates samples.

obtain here are in accordance with that of Holmes et al. (2004) and Hong \& Gu (2009), in both studies a maximum current was achieved within 10-20 days which was followed by a gradual decrease current production with time. The different intensity observed in current peak at different external load occurred as a result of higher catalytic activity on the bioanode at lower loads.

\subsection{Organic matter (OM) removal}

The removal efficiencies of soil OM increased with decreasing external resistance. The removal efficiency of $\mathrm{OM}$ in the vicinity of the bioanode were $17.7 \%$, $19.7 \%, 22.6 \%, 24.6 \%$ and $25.1 \%$ for the control, 2000 ohms, $1000 \mathrm{ohms}, 200 \mathrm{ohms}, 80 \mathrm{ohms}$ and $50 \mathrm{ohms}$ sMFC, respectively. These finds indicates that external resistance can influence OM removal efficiency (Song et al., 2010).

\subsection{Change in total iron and arsenic}

The iron and As concentration decreased with decreasing external resistance in the bioanode vicinity. Figure 1a-d illustrates the transformation of As and iron in different treatment over the course of the 90 days of incubation. As shown in Figure 1a and b, when sMFC was applied the iron concentration in in all of the treatments to a maximum on day 20 and then gradually declined until the end of the experiment. Arsenic concentration in soil pore water followed a similar trend (Figs. 1c and 1d). At the end of the experiment lower iron and As concentrations were observed in all treatments in the vicinity of the bioanode compared to the control. Furthermore, treatments with lower external resistance $(50,80$ and $200 \mathrm{ohms})$ had significantly lower soil pore water iron and As concentration compared with those at higher external resistance (1000 and $2000 \mathrm{ohms}$ ).

However in the bulk soil $2 \mathrm{~cm}$ away from the bioanode slightly higher iron and As concentration was observed in the treatments compared to control group. This suggests in the vicinity of the bioanode iron reduction and As mobility in the anoxic paddy soil was limited. This occurred as a result of increase OM removal by the bioanode.

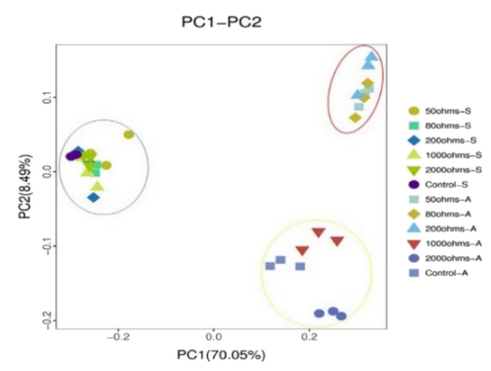

Figure 2. Principal Coordinates Analysis (PCoA) of the sMFC and controls bacterial community composition based on the unweighted UniFrac distance matrix.

\subsection{Microbial community}

The Illumina high through-put sequencing technique was used to investigate the influence of the different external resistance on the microbial community structure. Results shows that the external resistance applied can alter the microbial community that develop in the bioanode associated soil, but had minor effect on the bulk soil community (Fig. 2).

\section{CONCLUSIONS}

The results obtained in this study demonstrated that operating sMFCs at different external resistance can influence: i) The soil organic matter removal efficiency; ii) The iron and As release in anaerobic paddy soil; and iii) the microbial community that develop on the bioanode.

\section{ACKNOWLEDGEMENTS}

This work was supported by the National Science Foundation of China (41571305).

\section{REFERENCES}

Bond, D.R., Holmes, D.E., Tender, L.M. \& Lovley, D.R. 2002. Electrode reducing microorganisms that harvest energy from marine sediments. Science 295(5554): 483-485.

Fitzgerald, M.A., Mccouch, S.R. \& Hall, R.D. 2009. Not just a grain of rice: the quest for quality. Trends Plant Sci. 14(3): 133-139.

Holmes, D.E., Bond, D.R., O’Neil, R.A., Reimers C.E., Tender, L.R. \& Lovley, D.R. 2004. Microbial communities associated with electrodes harvesting electricity from a variety of aquatic sediments. Microb. Ecol. 48(2): 178-90.

Hong, Y.G. \& Gu, J.D. 2009. Bacterial anaerobic respiration and electron transfer relevant to the biotransformation of pollutants. Int. Biodeter Biodegr. 63(8): 973-980.

Song, T. S., Yan, Z.S., Zhao, Z.W. \& Jiang, H.L. 2010. Removal of organic matter in freshwater sediment by microbial fuel cells at various external resistances. $J$. Chem. Technol. Biot.

Xu, B.J., Ge, Z. \& He, Z. 2015. Sediment microbial fuel cells for wastewater treatment: challenges and opportunities. Environ. Sci. Water Res. Technol. 1(3): 279-284. 